\title{
Simple heuristics and the modelling of crowd behaviours
}

\author{
Mehdi Moussaïd $^{1 *}$, and Jonathan D. Nelson ${ }^{1}$ \\ ${ }^{1}$ Center for Adaptive Behavior and Cognition, Max Planck Institute for Human Development, \\ Lentzeallee 94, 14195 Berlin, Germany \\ ${ }^{*}$ Correspondence may be addressed to moussaid@mpib-berlin.mpg.de
}

\begin{abstract}
A crowd of pedestrians is a complex system that exhibits a rich variety of selforganized collective behaviors, such as lane formation, stop-and-go waves, or crowd turbulence. Understanding the mechanisms of crowd dynamics requires establishing a link between the local behavior of pedestrians during interactions, and the global dynamics of the crowd at high density. For this, the elaboration of a model is necessary.

In this contribution, we will make a distinction between two kinds of modelling methods: outcome models that are often based on analogies with Newtonian mechanics, and process models based on concepts of cognitive science. While outcome models describe directly the movements of a pedestrian by means of repulsive forces or probabilities to move from one place to another, process models generate the movement from the bottom-up by describing the underlying cognitive process used by the pedestrian during navigation.

Here, we will describe and compare two representatives of outcome and process models, namely the social force model on the one hand, and the heuristic model on the other hand. In particular, we will describe the strength and the limitations of each approach, and discuss possible future improvements for process models.

Keywords: Outcome models - Process models - Pedestrian behaviour - Crowd dynamics - Complex systems - Social forces - Simple heuristics
\end{abstract}

\section{Introduction}

Human crowds display a rich variety of collective behaviors that support an efficient motion under everyday conditions (1). For example, when two flows of people are moving in opposite directions in a crowded street, pedestrians spontaneously share the available space by forming lanes of uniform walking directions (2-4). This apparent "highway of pedestrians" is a decentralized collective organization that enhances the traffic efficiency by reducing the need for avoidance maneuvers among individuals. As another example, when two opposite flows meet at a narrow bottleneck, each flow temporarily "captures" the doorway during a short time period, resulting in alternating bursts of pedestrians passing first in one direction and then in the other. Again, this spontaneous group coordination allows for efficient usage of congested areas (5). 
The combination of local interactions among pedestrians, however, does not always generate efficient traffic solutions at the group level. For example, it has been shown recently that above a critical density level the collective coordination may suddenly break down, giving way to a dangerous phenomenon called crowd turbulence, in which the flow of pedestrians becomes chaotic (6). This particular regime is often observed during crowd disasters and is characterized by random and unintended displacements of people in all possible directions. The emergence of stop-and-go waves is another example of collective traffic perturbation that emerges spontaneously out of local interactions among pedestrians (6).

The key element for understanding the wide variety of collective behaviours observed in crowds lies in the different natures of local interactions among individuals (7). A local interaction is any kind of social influence that motivates an individual to change or adapt his or her behaviour based on social cues originating from neighbouring individuals. In pedestrian crowds, one may distinguish at least five different types of interactions:

- Collision avoidance is the basic and most common interaction among pedestrians. It describes the strategic adaptation of walking speed and direction to avoid an upcoming collision with another person. Collision avoidance is at the origin of the lane formation phenomenon in bidirectional flows, and also gives rise to stop-and-go waves in unidirectional flows at intermediate density.

- Physical interaction takes place when people are in physical contact with one another, at high density levels. Unlike collision avoidance that is based on intentional navigation strategies driven by visual information, physical interaction results in unintentional movements based on pushing and physical pressures exerted among densely packed people. Physical interaction is typically involved in the emergence of crowd turbulence.

- Social interaction drives the behaviour of social groups of pedestrians, such as friends going together to the same place. In social interaction, group members not only try to avoid collisions with one another, but also try to stay together, and often to converse with other group members. Social interactions are at the origin of the walking patterns observed in small groups of pedestrians and may reduce overall traffic efficiency (8).

- Imitation is another kind of interaction that takes place when people modify their walking destination to move in the same direction as other surrounding individuals. Imitation is assumed to have a major role during escape panics and to give rise to herding behaviour and unbalanced exit usage (9).

- Finally, we may also mention indirect interaction, wherein people adapt their walking behaviour not directly based on their neighbours' behaviour, but on the traces left in the environment by other pedestrians who are not present any more. This is the case, for example, when people walk over the trails left by others over green places or snowy areas (10).

Understanding the link between local interactions and emerging collective patterns is among today's most interesting challenges in the study of crowd behavior. For this, it is necessary to find a proper description of how pedestrians adapt their walking 
behaviour during each kind of interaction, and to explore the resulting collective patterns through computer simulations.

Nevertheless, finding a realistic description of pedestrians' behaviour is surprisingly challenging. Collision avoidance, for example, is a seemingly very complex cognitive task that requires collecting information about the location, the movements and the expected behaviours of other people, processing this information in real time, and planning a new trajectory every time the environment changes. Despite its apparent cognitive complexity, people easily perform this task in everyday life, even without being fully focused on the problem. We wish to distinguish two possible approaches to describe the pedestrian behaviour:

1. Elaborate an outcome model that directly describes the movements of the pedestrian based on complete information available in the environment. The social force model, for example, is an illustration of such an approach, in which the movements of people are described by means of repulsive and attractive forces.

2. Elaborate a process model that describes the underlying cognitive process that give rise to the movement. Process models typically aim at reproducing how the pedestrian processes a large amount of visual information to adapt his or her movement in real time. The heuristic model that we discuss below is an example of such an approach.

In this article, we will first describe an outcome model and discuss its strengths and limitations. Then, we will define more precisely the distinction between outcome models and process models. Finally, we will describe a process model of pedestrian behaviour, compare both approaches, and discuss its possible future improvements. Throughout the paper, we will focus mainly on two kinds of interaction, because these are responsible for the major part of collective crowd behaviours: collision avoidance and physical interaction.

\section{$2 \quad$ Modelling}

The process of modeling consists of finding a good description of the behavior of a pedestrian in his social and physical environment. A great deal of current research is devoted to the modeling of crowd behavior. In particular, a relevant model should be able to predict the emergence of observed crowd behavior during computer simulations. The necessity of elaborating a good and reliable model is twofold:

- First, the model constitutes an essential research tool to understand the precise mechanisms underlying the emergence of collective patterns. For instance, a model can be used to grasp why a particular pattern emerges, under which conditions, and which behavioral variables affect its features.

- Second, the model is also an important planning tool from an applied perspective. It can be used by urban planners to anticipate the behavior of crowds in a given environment, and help them manage events with large groups of people, the planning of evacuation strategies, or the assessment of urban layouts. 
Many current models of pedestrian behavior are outcome models based on analogies with physical systems. The main idea of this family of models is to suppose that the movements of a pedestrian in the crowd could be described by means of mathematical tools and theoretical concepts previously elaborated to describe the movements of a particle in a gas. Force-based models inspired from Newtonian mechanics are probably the most dominant in the scientific literature, and the most commonly used for the development of commercial modeling software.

In the following section, we will first focus specifically on force-based models, describe their basic underlying principles and discuss the strengths and limitations of this approach.

\subsection{Force-based modeling framework}

The so-called social force model was probably the first individual pedestrian-based model that was capable of predicting the emergence of collective, self-organized crowd patterns out of local interactions among individuals $(11,12)$. Since its first publication in 1995, there has been important development of this model and new models based on similar concepts $(13,14)$. Therefore, the original social force model can be seen today as a general framework commonly used in the community of crowd modelers.

The basic ingredients of the social force framework describe the motion of a pedestrian $i$ at place $\vec{x}_{i}(t)$ by means of a vector $\vec{F}_{i}$, reflecting his or her movement in a particular direction. Accordingly, the velocity $\vec{v}_{i}(t)=d \vec{x}_{i} / d t$ of pedestrian $i$ is given by the acceleration equation $d \vec{v}_{i}(t) / d t=\vec{F}_{i}(t)+\vec{\varepsilon}(t)$, where $\vec{\varepsilon}(t)$ is a fluctuation term that takes into account random variations of behavior. The acceleration force $\vec{F}_{i}$ is the core element of this modeling approach. It is defined as the sum of several terms denoting different attractive and repulsive effects, such as moving toward a destination point, avoiding static obstacles and other pedestrians, or staying close to other group members. In its simplest specification, the acceleration force includes three components:

- A driving force, $\vec{D}_{i}$, which lets the pedestrian $i$ move in his or her desired direction $\vec{e}_{i}$ at the desired speed $v_{i}^{o}$. The driving force is set such that the pedestrian adjusts the current velocity $\vec{v}_{i}$ to the desired one $v_{i}^{o} \vec{e}_{i}$, within a certain relaxation time $\tau_{i}$. This implies $\vec{D}_{i}=\left(v_{i}^{o} \vec{e}_{i}-\vec{v}_{i}\right) / \tau_{i}$. In recent experimental research, it has been shown that this equation indeed correctly reproduces the motion of a single pedestrian walking in a corridor, having the relaxation time $\tau_{i}=0.54$ seconds 
- A set of repulsive forces $\sum_{j \neq i} \vec{R}_{i j}$, which makes pedestrian $i$ avoid other pedestrians $j$ by moving away from them. In its simplest form, the term $\vec{R}_{i j}$ is defined as a gradient of a repulsion potential, resulting in $\vec{R}_{i j}=A_{i} e^{-d_{i j} / B_{i}} \vec{d}_{i j}$, where $\vec{d}_{i j}$ is the normalized vector pointing from $j$ to $i$, and $d_{i j}$ is the distance between the pedestrians; $A_{i}$ and $B_{i}$ are model parameters reflecting the strength and the range of the interaction, respectively. Nevertheless, many other specifications have been suggested (e.g. 9, 14, 15). Because it reflects how one individual reacts during interactions with other people, this component is the key element determining how the pedestrian behaves in the crowd.

- A set of repulsive forces $\sum_{k} \vec{W}_{i k}$, which makes pedestrian $i$ to keep a certain distance from walls and physical obstacles $k$ of the environment. The influence of an obstacle $k$ is defined as a function of the distance $d_{i k}$ to the closest point of that obstacle: $\vec{W}_{i k}=A_{k} e^{-d_{i k} / B_{k}} \vec{d}_{i k}$, where $\vec{d}_{i k}$ is the normalized vector pointing from $k$ to pedestrian $i, A_{k}$ and $B_{k}$ are model parameters.

While the very first specifications of the social force model had only qualitative connections to empirical observations, many recent studies made use of tracking algorithms to reconstruct trajectories of interacting pedestrians from video recordings taken in streets, train stations, or highly crowded areas, and to elaborate and calibrate increasingly precise specifications of the model (14-17). Today, the most recent specifications are able to generate realistic crowd behavior, and to reproduce - at least qualitatively - every known crowd behavior, including lane formation, oscillation at bottlenecks (11), social groups' behavior (8), stop-and-go waves (18), and crowd turbulence (19).

\subsection{Strength and limitations of physics-based approaches}

One of the greatest advantages of force-based models is their versatility, which probably explains the large variety of existing specifications in the literature. In fact, the social force framework is very easy to adapt by adding new parameters, changing their values, modifying the shape of the equation the forces involved, or adding new attractive or repulsive components. For example, many different specifications of the model only differ in the formulation of the equation of the main repulsive force $\vec{R}_{i j}$. Therefore, it is not difficult to extend the model to new kinds of interactions by adding a new term to the final equation. Thus, social interactions can be implemented by adding attraction forces among group members (8), indirect interactions with the active walker model (10), or imitation by means of a herding force in situations of panics (9). Another important strength of the social force framework is that it benefits from all the existing tools of statistical physics, which favors its development in the 
literature. For example, the transformation of the individual-based model into a macroscopic description based on partial differential equations is facilitated.

Nevertheless, the force-based approach exhibits some theoretical limitations. First, although force-based models can reproduce any empirical observation, it is important to notice that this often requires a modification, an adjustment, or a recalibration of the model. In fact, force-based models have excellent fitting capabilities, in the sense that a model can reproduce almost any observation after adjusting the equation of motion accordingly. However, they offer relatively low predictive power: a model that has been previously calibrated on a given dataset often fails to quantitatively predict another set of observations. One example is the experimental specification of the model presented in ref. (15). This force-based model was specifically designed to reproduce individual avoidance trajectories as observed under experimental conditions (we call it dataset A). However, it failed to quantitatively reproduce the complex dynamics of lane formation observed in ref. (3) (dataset B). Nevertheless, a recalibrated model finally allowed to reproduce correctly the dataset $\mathrm{B}$, but did not work any longer for dataset A. Therefore, this particular specification was not able to reproduce the features of dataset A and dataset B at the same time. Likewise, the recent simulation of crowd turbulence required the use of very strong inter-individual repulsive forces that would probably not be adapted for the simulation of pedestrian movements at low density (19). Thus, even through this specification is an extremely valuable tool for the planning of mass events where crowd turbulence often occurs, it implies using different specifications of the model depending on the expected crowd density level.

Another issue with force-based models is that quantitative agreement with empirical data often requires rather sophisticated movement equations. In particular, it seems from the past decade that crowd models have tended to become increasingly more complex in an attempt to reproduce the growing amount of new empirical observations. The specification presented in ref. (15), for instance, is a good illustration of how sophisticated the model equations have to be to precisely reproduce individual avoidance trajectories. In fact, it is not a trivial problem to find a proper balance between attractive and repulsive effects that would correctly reproduce the speed and direction changes during an avoidance manoeuver. In ref. (15), this was achieved at the cost of using sophisticated equations of motion, which is also the case for many other force-based specifications. Although the high complexity of a model does not undermine its simulation capabilities, it might reduce the model's usefulness for deciphering the dynamics of the system.

\subsection{Outcome models and process models}

One possible reason explaining the above limitations is that force-based models aim at describing directly the observed movements of pedestrians, rather than the internal cognitive processes leading to the observed movement. In other words, forcebased models are excellent at reproducing observations under specific conditions, but 
do not capture the intrinsic cognitive mechanisms operating at the level of the individual. It is very unlikely that pedestrians would be really under the influence of attractive and repulsive forces. Instead, it seems more plausible that they rely on simple and unconscious rules and navigation strategies, which let them act as if they were subject to external forces.

This is the distinction between so-called "outcome models" describing directly the observed behaviour, and "process models" describing the cognitive processes that give rise to the observed behaviour $(20,21)$. To illustrate the difference between outcome models and process models, consider the following example: Imagine the problem of modelling the movements of a baseball outfielder trying to catch a ball in the air. One can imagine two distinct modelling approaches. The first consists in measuring the expected landing point of the ball according to its current trajectory and other environmental variables, and to use an attraction force to describe the player's motion toward that point. Even through this modelling approach would require sophisticated equations and the use of many parameters, it would probably reproduce the movements of the player in a realistic manner. The second method is to elaborate a process model that relies on the so-called gaze heuristic - a simple rule-of-thumb that is used by people and animals to catch a moving target $(20,22)$. The gaze heuristic consists of three steps: Fix your gaze on the ball, start running, and adjust your running speed and direction so that the angle of gaze remains constant. A simple model implementing these three rules would also reproduce the movements of the player, but it can ignore all causal variables necessary to compute the landing point of the ball, such as the velocity, angle, air resistance, or speed and direction of wind among others. Interestingly, both methods would probably predict a similar trajectory. Nevertheless, the process model generates the motion from the bottom-up, and is easier to formulate than the outcome model.

The above example can be easily put in parallel with the modelling of pedestrian movements. Existing force-based models are outcome models. Yet, a consistent framework for the development of a process model is still lacking. In the following, we describe a first attempt to elaborate such a framework based on ref. (23), and discuss its strength and limitations.

\subsection{A process model of pedestrian behaviour}

The elaboration of a process model requires addressing and answering two key questions:

(1) What kind of information does the pedestrian use?

(2) How is this information processed?

To answer the first question, there is no doubt that a pedestrian relies on visual cues as primary source of information to decide where to walk $(24,25)$. Similar vision-based approaches to describe people's movement in complex buildings have been undertaken in the past (26-29). While interactions among individuals are the most important for the study of crowd dynamics, these studies only concern interactions between the individual and the physical environment. Recently, there have been several attempts 
to elaborate models of pedestrians' behaviour in crowds based on visual cues (30), among which is the heuristic model that we will present in the next section.

\section{Formalization of visual cues.}

What would be the simplest way to describe the visual cues that a pedestrian uses to navigate in a dynamic environment? Some simplifications have to be made here, as it would be impossible to describe the entire complexity of the human visual system. Therefore, we assume that the relevant information is the distance before collision with surrounding people, objects and obstacles. Therefore, we describe the input information of a pedestrian $i$ by a function $f(\alpha)$ describing the distance that the pedestrian can walk toward the direction $\alpha$ at his or her comfortable walking speed $S_{i}^{0}$ before a collision occurs. Because the function $f(\alpha)$ describes the visual information of the pedestrian, it is only defined for values of $\alpha$ in the interval $[-\phi, \phi]$ delimiting the vision range of the individual. Here, the value $\alpha=0$ corresponds to the looking direction represented by a unit vector $\vec{H}_{i}$ and the vision field of the pedestrian is $\phi$ degrees to the left and to the right of the direction $\vec{H}_{i}$. If no collision is found toward the direction $\alpha$, then the value of $f(\alpha)$ is set to a default maximum value $d_{\max }$, which represents the horizon distance of the pedestrian as sketched in Figure 1. Even though the computation of the collision distances with other people requires complex geometrical calculations, it remains a reasonable assumption as collision anticipation is a core capacity of the human brain $(24,31)$. It is important to note here that this formulation requires some other simplifying assumptions, such as the fact that pedestrians have a circular body of radius $r_{i}$ (although it is possible to adapt the model to elliptical body shapes as we will mention later in this article, in the 'Possible improvements' section).

\section{Two simple heuristics and body contacts.}

The second question that needs to be answered concerns the processing of this visual information. Having described the surrounding visual information, we need now to define a set of rules describing how the pedestrian exploit visual cues to navigate toward a destination point $\mathrm{O}_{i}$. For this, we assume that the pedestrian relies on two simple cognitive procedures, called heuristics (20). The first one delineates how the pedestrian adapts their direction of motion according to the occupancy of the visual field, and the second describes how the pedestrian adapts their walking speed as a function of the available walking space.

Minimizing detours. 
The first movement heuristic concerns the relative angle $\alpha_{d e s}$ of the chosen walking direction. Empirical evidence suggests that pedestrians seek an unobstructed walking direction, but dislike deviating too much from the direct path to their destination $(32,33)$. A trade-off therefore has to be found between avoiding obstacles and minimizing detours from the most direct route. Accordingly, our first heuristic is defined as follows:

"The chosen walking direction $\alpha_{\text {des }}$ is the one that minimizes walking towards the destination point."

In this way, the pedestrian chooses the unobstructed direction that deviates least from the destination point. Formally, the chosen direction $\alpha_{d e s}(t)$ is computed through the minimization of the distance to destination $d(\alpha)$ :

$$
d(\alpha)=\sqrt{d_{\max }^{2}+f(\alpha)^{2}-2 d_{\max } f(\alpha) \cos \left(\alpha_{0}-\alpha\right)} .
$$

Here, $\alpha_{0}$ is the direction of the destination point.

Keeping a safe distance.

The second heuristic determines the desired walking speed $v_{\text {des }}(t)$. Since a reaction time $\tau$ is required for the pedestrian to stop in the case of an unexpected obstacle, pedestrians should compensate for this delay by keeping a safe distance ahead (34). Therefore, we formulate the second heuristic as follows:

"The pedestrian reduces the walking speed to keep the time to collision with the nearest obstacle above $\tau$ seconds."

Formally, the desired speed is given by:

$$
v_{\text {des }}(t)=\min \left(v_{i}^{0}, d_{h} / \tau\right) \text {, }
$$

where $d_{h}$ is the distance between pedestrian $i$ and the first obstacle in the desired direction $\alpha_{d e s}$ at time t.

Finally, the vector $\vec{v}_{\text {des }}$ of the desired velocity points in direction $\alpha_{d e s}$, and has the norm $\left\|\vec{v}_{\text {des }}\right\|=v_{\text {des }}$. The change in the actual velocity $\vec{v}_{i}$ at time $t$ under normal walking conditions is given by the acceleration equation $d \vec{v}_{i} / d t=\left(\vec{v}_{\text {des }}-\vec{v}_{i}\right) / \tau$, where $\tau=0.5 \mathrm{~s}$ as determined in previous experimental measurements (15), and the location $\vec{x}_{i}(t)$ of pedestrian $i$ at time $t$ is the first derivative of the velocity $d \vec{x}_{i} / d t=\vec{v}_{i}$. The looking direction $\vec{H}_{i}$ is constantly adjusted toward the desired direction $\alpha_{d e s}$. 


\section{Pushing behavior.}

The above model has very good simulation performance at low density. For instance, it quantitatively reproduces the features of individual avoidance trajectories (23), as well as the emergence of unstable dynamics during lane formation (3). However, simulation results at higher densities are not satisfying. The reason for this is that the nature of interactions involved is different. In fact, when the density is high enough, physical contacts may occur as well, causing unintentional movements that are not determined by the above heuristics. Therefore, it is necessary to distinguish between intentional avoidance behaviors based on strategic navigation heuristics, and unintentional movements resulting from pushing forces caused by body contacts. Hence, it is necessary to extend the above description by considering a physical contact force that applies only when people are in physical contact:

$$
\vec{f}_{i j}=k g\left(r_{i}+r_{j}-d_{i j}\right) \vec{n}_{i j}
$$

where the sub-function $\mathrm{g}(\mathrm{x})$ is zero if the pedestrians $i$ and $j$ do not touch each other, and otherwise equals the argument $x$. Here, $\vec{n}_{i j}$ is the normalized vector pointing from pedestrian $j$ to $i$, and $d_{i j}$ is the distance between the pedestrians' centers of $\operatorname{mass}(9)$.

The final model allows for simulations of crowd movements at any density level, and is capable of quantitatively reproducing a large range of phenomena, such as the fundamental diagram, stop-and-go waves, some features of crowd turbulence, and evacuation times (23). In contrast to force-based approaches, the repulsive interaction term $\vec{f}_{i j}$ used in the cognitive model is not an analogy, but reflects the physical pressure among bodies that actually takes place in crowded environment. It is interesting to note that most existing models in the literature do not make a clear distinction between physical interactions and collision avoidance interactions, trying to describe both of them with the same tool. But these two kinds of interaction arise from fundamentally different processes: the physical pressure on the one hand, and the navigation strategy on the other hand.

\subsection{Benefits and drawbacks of the heuristic model}

Beside the fact that the heuristic model is based on plausible hypotheses and offers a new perspective on existing literature, it is interesting to note that it actually solves a long-standing open issue about how to combine multiple simultaneous interactions (35). For example, in a situation where an individual $\mathrm{A}$ is facing three other individuals $\mathrm{B}, \mathrm{C}$, and $\mathrm{D}$, force-based models typically assume that $\mathrm{A}$ sums up the repulsive effects that B, C and D would have separately in the absence of the others. However, this approach raises several theoretical issues. First, it is not obvious that simultaneous influences are additive. One can also integrate them in different ways such as averaging over them, or combining them nonlinearly. Second, it is unclear how to determine the influential neighbors and how to weight their influence. For example, it has been suggested that only the $k$ closest individuals should be taken into account, or 
only those located in a certain interaction radius $R$ (35-37). But the values of $k$ and $R$ remain unclear and seem to vary with increasing density level. Another subsequent problem is that the repulsive effect in force-based models is necessarily proportional to the number of interacting partners. Consider the example in which a person is avoiding a group of standing pedestrians. Because repulsive forces typically cumulate, the shape of the avoidance trajectory will change according to the number of people in the standing group, which often generates inconsistent simulations. In contrast, in the heuristic model, interactions cannot be reduced to the superimposition of pair interactions because individuals react to a visual pattern in an integrative way. Therefore, rather than balancing multiple repulsion effects originating from other individuals, the simulated pedestrian detects an efficient free way to the destination and follows it, which generates realistic behaviour from the bottom-up. Therefore, it is not necessary to define explicit rules for combining simultaneous interactions or setting a fixed number of interacting partners in advance. These problems vanish naturally when using an integrated visual input, such as the pedestrian's visual field.

Nevertheless, the cognitive model exhibits technical limitations due to the difficulty of its implementation. In fact, the calculation of the function $f(\alpha)$ describing the visual pattern of the pedestrian is a complex algorithm that requires heavy computational power and is prone to programming errors. The program typically needs to identify the first collision point between one moving object (the focus pedestrian) and many others (the other individuals), and to repeat this step for all directions $\alpha$ of the vision field, for all pedestrians $i$, and at every time step $t$. Therefore, simulations of the cognitive model are systematically longer than simulations of a force-based model, and may reach unreasonable computation time for simulations of large crowds (e.g. higher than 1000 individuals). Even through this technical limitation does not directly challenge the validity of the model, it can hinder or slow down its future development, and dissuade the use of the model in commercial software. In the next section, we will suggest the search-and-stop rule as a future improvement that is likely to reduce the needed computational power. Advances in programming techniques and particularly in ray-casting algorithms may also help in resolving this issue.

Another issue with the cognitive model is that it is more difficult to extend than forcebased models. With the social force approach, modeling a new component of pedestrians' behavior, such as the side preference, the herding behavior during panics, or the movements of pedestrians in social groups, always consists of adding a new force component and calibrating this force with empirical data $(8-10,15)$. This systematic methodology facilitates the development of the model. Updating the cognitive model, however, always requires some deep questioning about the cognitive processes involved in the situation being studied. For example, the cognitive model fails to correctly reproduce the situation where two pedestrians are facing each other at a narrow bottleneck, such as a door. In this situation, the visual field of each individual indicates that there is no empty space where it is possible to move, and therefore both pedestrians stop moving and wait endlessly for an opportunity to move ahead. Of course, this situation would never happen in real life because people have learnt that 
one has to step back and let the other one go first. But implementing this improvement in the model is more complicated, as simulated pedestrians need to identify the problem first, and solve it afterward.

\subsection{Possible improvements}

As we mentioned earlier in this article, the cognitive approach described above should be considered as an initial basic framework rather than a final model. We have deliberately kept the model in its simplest form so far, to highlight the most basic heuristics needed to reproduce the fundamental features of crowd dynamics. But this first attempt certainly requires many improvements. In this section, we describe some possible improvements that could be considered in the future.

The first modification that could be undertaken is the implementation of elliptical body shape. Although the circular body shape seems to be a reasonable simplification in the first place (as demonstrated by the accurate predictions of the model), it may be relevant to use an elliptical body shape instead in the future (38). In fact, at high densities, elliptical bodies may reduce the collision frequency and therefore affect the density threshold at which stop-and-go waves and crowd turbulence form. The implementation of elliptical body shape would also constitute a first step for modeling movements of pedestrians' shoulders, which is known to facilitate avoidance maneuvers in crowded areas (39). This improvement is expected to be important even at low density because it may help unblocking some face-to-face situations, such as the one described in the previous section. However, this update remains challenging, as it will require even more sophisticated algorithms for the detection of collisions.

Another important improvement would be the elaboration of a search-and-stopping rule $(20,40)$. The current version of the model presumes that pedestrians select the best walking direction among all directions within their field of view (i.e. the direction that minimizes the distance to the destination point). However, it might be more realistic to assume that the pedestrian instead searches a good walking direction in his visual pattern and stops searching when a good enough solution is found, without exploring the complete vision field. This would require the implementation of a search rule (what is the starting point of the searching? What is the exploration sequence?), and a stopping rule (what is a good enough solution? When to stop searching?). Ideally, this rule would predict the kinds of eye movements that pedestrians engage in, based on theoretical principles and empirical data (41). For example, one may assume that the individual starts searching in front of him (around $\alpha=0$ ), explores the visual pattern toward the sides, and stops when a satisficing walking direction is found. In addition, biased exploration patterns toward the right-hand side could possibly reproduce the features of the side preference (15). The search-and-stop rule could also drastically reduce the computational power, as the complete field of vision would not need to be computed in advance, and might produce approximate decisions in a way that is closer to the pedestrian's actual decision process. 
Finally, it could also be interesting to include noise and fluctuations in the simulated behavior of the pedestrian. In particular, most existing modeling approaches rely on an "extrinsic" source of fluctuations, in the sense where random fluctuations of the movement are added after the deterministic behavior has been calculated. In force-based models, for instance, the equation of movement typically includes a random component that causes the pedestrian to deviate randomly from a precalculated position (e.g. the term $\vec{\varepsilon}(t)$ in the original equation of the social force model).

When using a heuristic-based approach, however, it becomes possible to inject an intrinsic source of stochasticity. In such a way, the noise is not added after the movement is computed, but directly included in the cognitive process that generates the movement. For example, one may add random errors when the pedestrian estimates the expected collision points with other pedestrians, with less precise estimations on the sides than in the center of the visual field. The precision of the estimates in the model could even be based on data on the relative area that the visual cortex devotes to processing stimuli at a particular distance from the center of the visual field (42). When the pedestrian makes eye movements (fixations) to particular parts of the visual field, the precision of their estimates of the location and behavior of stimuli near that part of the visual field would increase the most.

\section{Conclusion}

In this article, we have described and compared two modelling frameworks for simulating crowd dynamics. While an outcome model can make use of attractive and repulsive forces to describe a pedestrian's movements, a process model based on concepts of cognitive science describes the internal processes underlying the movement. We underlined the fact that different kinds of interaction, namely collision avoidance and physical interactions, should be described with different tools that account for the specific nature of each type of interaction: simple heuristics to describe the pedestrian's navigation strategy, and a repulsive force to account for body contacts in dense crowds.

When comparing the cognitive approach to the classical social force framework, it seems that each method complements the other, and has its own benefits and drawbacks. Each approach has similar performance in simulations, but the cognitive approach offers the greater potential for connecting pedestrian decision processes with individual and aggregate pedestrian behaviour. Nevertheless, we believe that outcome- and process-based models, by existing in parallel, will facilitate the development of comprehensive theories of crowd dynamics that could not arise from either approach alone. 
1. Helbing D, Molnar P, Farkas IJ, Bolay K (2001) Self-organizing pedestrian movement. Environment and Planning B: Planning and Design 28:361-383.

2. Yamori K (1998) Going with the flow: Micro-macro dynamics in the macrobehavioral patterns of pedestrian crowds. Psychological review 105:530-557.

3. Moussaïd M et al. (2012) Traffic Instabilities in Self-Organized Pedestrian Crowds. PLoS Comput Biol 8:e1002442.

4. Kretz T, Grünebohm A, Kaufman M, Mazur F, Schreckenberg M (2006) Experimental study of pedestrian counterflow in a corridor. Journal of Statistical Mechanics: Theory and Experiment 2006:P10001-P10001.

5. Helbing D, Buzna L, Johansson A, Werner T (2005) Self-Organized Pedestrian Crowd Dynamics: Experiments, Simulations, and Design Solutions. Transportation Science 39:1-24.

6. Helbing D, Johansson A, Al-Abideen H (2007) The Dynamics of crowd disasters: an empirical study. Physical Review E 75:46109.

7. Camazine S et al. (2001) Self-Organization in Biological Systems (Princeton University Press).

8. Moussaïd M, Perozo N, Garnier S, Helbing D, Theraulaz G (2010) The Walking Behaviour of Pedestrian Social Groups and Its Impact on Crowd Dynamics. PLoS ONE 5:e10047.

9. Helbing D, Farkas I, Vicsek T (2000) Simulating dynamical features of escape panic. Nature 407:487-490.

10. Helbing D, Keltsch J, Molnar P (1997) Modelling the evolution of human trail systems. Nature 388:47-50.

11. Helbing D, Molnar P (1995) Social force model for pedestrian dynamics. Physical Review E 51:4282-4286.

12. Helbing D (1991) A mathematical model for the behavior of pedestrians. Behavioral Science 36:298-310.

13. Yu WJ, Chen R, Dong LY, Dai SQ (2005) Centrifugal force model for pedestrian dynamics. Physical Review E 72:26112.

14. Johansson A, Helbing D, Shukla P (2007) Specification of the social force pedestrian model by evolutionary adjustment to video tracking data. Advances in Complex Systems 10:271-288.

15. Moussaïd M et al. (2009) Experimental study of the behavioural mechanisms underlying self-organization in human crowds. Proceedings of the Royal Society B: Biological Sciences 276:2755-2762.

16. Johansson A, Helbing D, Al-Abideen HZ, Al-Bosta S (2008) From crowd dynamics to crowd safety: A video-based analysis. Advances in Complex Systems 11:479-527.

17. Hoogendoorn S, Daamen W (2007) in Traffic and Granular Flow, pp 329-340.

18. Helbing D, Johansson A, Mathiesen J, Jensen M, Hansen A (2006) Analytical Approach to Continuous and Intermittent Bottleneck Flows. Physical Review Letters 97:168001.

19. Yu W, Johansson A (2007) Modeling crowd turbulence by many-particle simulations. Physical Review E 76:46105.

20. Gigerenzer G, Todd P (1999) Simple Heuristics That Make Us Smart (Oxford University Press).

21. Gigerenzer G, Brighton H (2009) Homo Heuristicus: Why Biased Minds Make Better Inferences. Topics in Cognitive Science 1:107-143.

22. Bennis W, Pachur T (2006) Fast and frugal heuristics in sports. Psychology of Sport and Exercise 7:611-629. 
23. Moussaïd M, Helbing D, Theraulaz G (2011) How simple rules determine pedestrian behavior and crowd disasters. Proceedings of the National Academy of Science 108:6884-6888.

24. Gibson J (1979) The Ecological Approach To Visual Perception (Houghton Mifflin).

25. Gibson JJ (1958) Visually controlled locomotion and visual orientation in animals. British Journal of Psychology 49:182-194.

26. Hillier B, Hanson J (1984) The Social Logic of Space (Cambridge University Press).

27. Garling T, Garling E (1988) Distance minimization in downtown pedestrian shopping. Environment and Planning A 20:547-554.

28. Penn A, Turner A (2002) in Pedestrian and Evacuation dynamics, eds Schreckenberg M, Sharma S (Springer), pp 99-114.

29. Turner A (2007) in 6th International Space Syntax Symposium, eds Kubat AS, Ertekin O, Guney YI, Eyuboglu.

30. Ondřej J, Pettré J, Olivier A-H, Donikian S (2010) A synthetic-vision based steering approach for crowd simulation. ACM Trans Graph 29:1-9.

31. Cutting JE, Vishton PM, Braren PA (1995) How we avoid collisions with stationary and with moving obstacles. Psychological Review 102:627-651.

32. Batty M (1997) Predicting where we walk. Nature 388:19-20.

33. Turner A, Penn A (2002) Encoding natural movement as an agent-based system: an investigation into human pedestrian behaviour in the built environment. Environment and Planning B: Planning and Design 29:473-490.

34. Johansson A (2009) Constant-net-time headway as a key mechanism behind pedestrian flow dynamics. Physical Review E 80:26120.

35. Ballerini $\mathrm{M}$ et al. (2008) Interaction ruling animal collective behavior depends on topological rather than metric distance: Evidence from a field study. Proceedings of the National Academy of Sciences 105:1232.

36. Steffen B (2008) in Conference proceedings of PED2008 (Springer, Berlin).

37. Ma J, Song W, Zhang J, Lo S, Liao G (2010) k-nearest-neighbor interaction induced self-organized pedestrian counter flow. Physica A 389:2101-2117.

38. Still K (2000) Crowd Dynamics.

39. Daamen W, Hoogendoorn S (2002) Controlled experiments to derive walking behaviour. Journal of Transport and Infrastructure Research 3:39-59.

40. Hutchinson J, Gigerenzer G (2005) Simple heuristics and rules of thumb: Where psychologists and behavioural biologists might meet. Behavioural Processes 69:97-124.

41. Nelson J, Cottrell G (2007) A probabilistic model of eye movements in concept formation. Neurocomputing 70:2256-2272.

42. Sereno MI et al. (1995) Borders of multiple visual areas in humans revealed by functional magnetic resonance imaging. Science 268:889-893. 


\section{$5 \quad$ Figure}
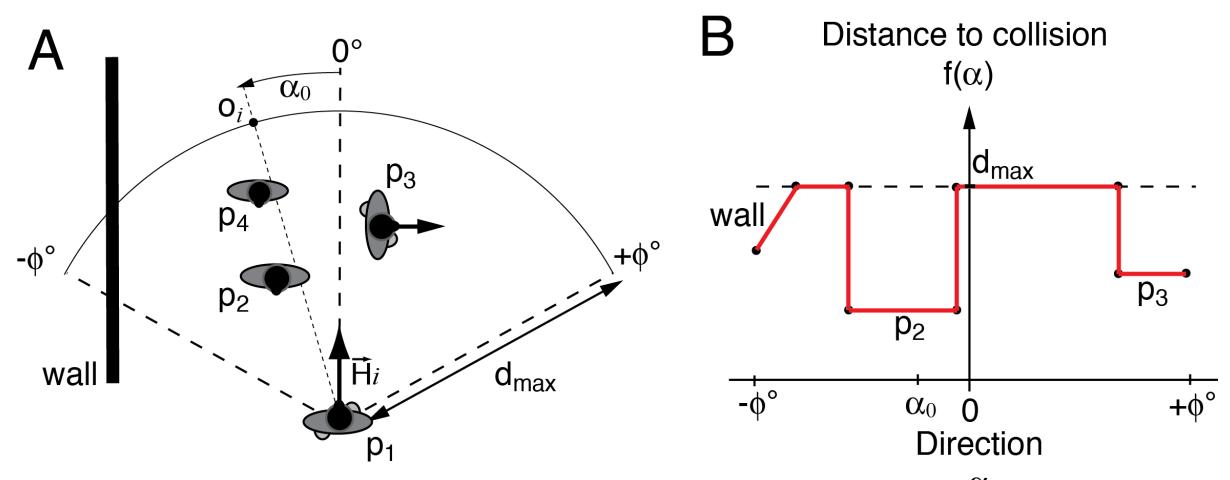

$\alpha$

Fig. 1. A) Illustration of a pedestrian $\mathrm{p}_{1}$ facing three other individuals and a wall, trying to reach the destination point $\mathrm{O}_{\mathrm{i}}$. B) Graphical representation of the function $f$ reflecting the distance to collision with any obstacle when moving into different directions. The wall covers the left-hand side of the vision field. Pedestrian $\mathrm{p}_{4}$ is not visible because it is hidden by $\mathrm{p}_{2}$. Pedestrian $\mathrm{p}_{3}$ is moving away, so a collision would occur only if $\mathrm{p}_{1}$ moves toward the right-hand side. 